\title{
Carpal tunnel syndrome in HIV-positive patients coinfected with HCV
}

\author{
A. Mastroianni' ${ }^{1}$, F. Allegrini' ${ }^{1}$, S. Nardi ${ }^{2}$, D. Donatucci' ${ }^{2}$, F. Girelli ${ }^{3}$, C. Guidi ${ }^{4}$ \\ 'Unità Operativa Malattie Infettive; ${ }^{2}$ Unità Operativa Ortopedia; ${ }^{3}$ Unità Operativa Medicina Interna - Reumatologia; \\ ${ }^{4}$ Unità Operativa Neurologia, Presidio Ospedaliero G. B. Morgagni - L. Pierantoni, Forli, Italy
}

\begin{abstract}
SUMMARY
A wide range of rheumatic and peripheral nervous system disorders may develop in patients with HIV infection, leading to pain, sensory symptoms, and muscle weakness. Over the past three decades, the progress in management of HIV disease with anti-retroviral therapy (ART) has resulted in increased life expectancy for people living with HIV disease. With this new chronicity of the disease has a constellation of chronic musculoskeletal, orthopaedic and rheumatic manifestations has emerged, as potential complications of the disease itself and/or the results of ART treatment regimen and/or because of expected age-related symptoms/manifestations. The incidence of CTS in the general population is around 3.8\% with clinical examination and, when electroneuromyography is used, it is $2.7 \%$. In the HIV-positive population, the incidence is very close to that of the general population. The aim of this study was to evaluate the incidence of CTS and to identify factors influencing the development of CTS in HIV-infected patients attending our clinic. This syndrome has been associated with advanced HIV disease and the use of ART possibly due to an increased inflammatory state and the presence of concurrent HCV infection.
\end{abstract}

Key words: Carpal Tunnel Syndrome; HCV; HI; HAART; ART; Peripheral Neuropathy, Autoimmunity; Extrahepatic disorders; HCV; Hepatitis; Hepatitis C virus; Mixed cryoglobulinemia; Peripheral Neuropathy.

Reumatismo, 2017; 69 (4): 164-169

\section{INTRODUCTION}

The considerable increase in the life 1 expectancy of HIV-infected patients in the age of highly-active antiretroviral therapy (HAART) results in a wide range of peripheral nerve diseases, metabolic and bone-joint changes resulting from a longlasting viral infection time and from its treatment (1).

In the HAART era, rheumatic complications declined significantly but continue to be prevalent with manifestations including the emerging of a new syndrome of immune reconstitution (2). The rheumatic and osteoarticular changes most frequently reported in patients infected for a long period with HIV and using ART are osteopenia/ osteoporosis, osteonecrosis, carpal tunnel syndrome (CTS), and adhesive capsulitis of the shoulders (1). The aim of this study was to evaluate the incidence of CTS and to identify factors influencing the development of CTS $(3,4)$, as well as results of its surgical treatment in patients with HIV infection attending our clinic. The study included 182 HIV-positive patients with peripheral neuropathies, among whom CTS was diagnosed in 12 patients on the basis of signs and physical symptoms, as well as by nerve conduction studies. We also conducted a comprehensive MEDLINE literature search of all reports concerning CTS in individuals with HIV infection published between 1981 and 2016.

\section{MATERIALS AND METHODS}

We retrospectively reviewed the records of HIV-infected patients managed in the Infectious Diseases Unit, Department of Internal Medicine of the Forlì Hospital, Italy, between January 1996 and December 2016. Therefore, the first phase of this retrospective analysis was to identify patients with rheumatic manifestations and to select patients with peripheral nervous system complications, in order to identify cases of CTS evaluated in a neurological and/or rheumatological and/or orthopaedic 
specialist setting. The cohort also included inmates who entered the Forlì correctional facility and were evaluated through referrals from health care providers. Immunovirological evolution, opportunistic infections, neoplasia occurrence, duration of HAART, and type of HAART at CTS diagnosis were recorded.

\section{RESULTS}

CTS history was characterized by date of diagnosis, immuno-virological context at diagnosis, clinical manifestations, treatments, complications of these treatments and outcome.

Out of 783 patients, we identified 182 (130 male, 52 female) patients with peripheral neuropathies; of these 182 patients, twelve patients had a diagnosis of CTS on the basis of signs and physical symptoms, as well as by nerve conduction studies.

The age range was 1-10 years earlier; HIV infection was diagnosed 1-10 earlier in these patients with CTS. Initial analysis of CTS incidence by sex, age, duration of HIV infection and ART, pregnancy, diabetes, thyroid disease, rheumatoid arthritis, injuries such as wrist fractures, repetitive work activities such as personal computer use and/or other repetitive job-related hand motions that can produce vibrations, and the presence of anti-HCV antibodies was undertaken. Duration of HIV disease and ART and HCV-positivity were the most important and significant risk factors for the development of CTS. No significant differences were found when we evaluated CTS incidence by sex. In fact, among patients with CTS, the incidence substantially overlapped in both sexes (5 males, 7 females). Patients with CTS were aged between 26 and 53 years, while the asymptomatic patient group was aged 19 to 72 years. ART in the patient group with CTS ranged from 4 to 15 years, while ART duration among patients without CTS ranged from 0.2 to 12.4 years.

Median CD4 T lymphocyte count at CTS diagnosis was $385 / \mathrm{mm}^{3}$. For the 9 patients who were treated with ART before the CTS diagnosis, median viral load at CTS diag- nosis was undetectable ( $<50$ copies $/ \mathrm{mL}$ ). For the 3 patients who did not have ART at CTS diagnosis, median viral load and median CD4 T lymphocyte count at CTS diagnosis were $224 \mathrm{~mm}^{3}$ and 22.570 copies/mL, respectively.

Seven patients had HCV genotype 1, 4 had genotype 2 and 1 had genotype 3 . Three patients were cirrhotic. HCV RNA quantity was significantly higher in patients with genotype 1 (mean, 1.233 copies $/ \mathrm{mL}$ ) than in patients with other genotypes (mean, 654.450 copies $/ \mathrm{mL}$ ).

All affected patients reported paresthesias and pain in the palmar side of the first three fingers of both hands, with sensory loss, and weakness on abduction. Every patient underwent bilateral median motor conduction studies evaluating distal latency, conduction velocity, and amplitude of compound motor action potentials. Bilateral median sensory nerves were also evaluated for distal latency, amplitude of response, and conduction velocity. Median motor and sensory studies in all patients revealed prolonged sensory latencies, and moderate to severe residual motor latencies.

In all cases a diagnosis of work-related carpal tunnel syndrome was excluded after an appropriate analysis. There was no evidence of opportunistic infections, metabolic abnormalities, or HIV lipodystrophy syndrome in all patients. In all cases there was no history of underlying medical conditions associated with an increased risk for the development of carpal tunnel syndrome, such as diabetes, pregnancy, rheumatoid arthritis, hypothyroidism, acromegaly, monoclonal gammathies or amyloidosis. All patients had concomitant mild to moderate HCV-related chronic viral hepatitis.

A variety of treatment regimens were used reflecting the evolution of HIV disease therapy in clinical practice. All but three female patients naïve for ART were treated with a protease inhibitor-based ART regimen. Surgical release procedure was required in 10 patients $(85 \%)$ with CTS on ART for a long period (from 10-15 years). Surgery was effective in most patients, but in two patients some symptoms persisted. 


\section{DISCUSSION}

AND CONCLUSIONS

Many rheumatic manifestations and several types of peripheral neuropathy may occur in HIV-infected persons and they may be either caused by the HIV infection itself, triggered by adaptive changes in the immune system, secondary to microbial infections, or from side-effects of ART.

HIV-infected individuals are at an increased risk of developing musculoskeletal pathology. Rheumatic diseases and asymptomatic immune phenomena remain prevalent in $\mathrm{HIV}$-infected persons even after the widespread implementation of highly active anti-retroviral therapy. $\mathrm{Pa}$ tients with AIDS may be particularly susceptible to developing nerve compression in the presence of malnutrition and weight loss or wasting syndrome; the most common entrapment neuropathies observed in malnourished AIDS patients are cubital tunnel syndrome due to ulnar nerve compression at the elbow producing pain and paraesthesias in the forearm and $4^{\text {th }}$ and $5^{\text {th }}$ digits, sometimes with weakness in the intrinsic hand muscles; common peroneal nerve compression at the fibula head resulting in a foot drop with numbness over the lateral lower extremity and dorsum of the foot; meralgia paraesthetica with entrapment of the lateral cutaneous nerve of the thigh at the inguinal ligament and paraesthesia over the lateral thigh and possibly a more diffuse neuropathy; and tarsal tunnel syndrome with entrapment of the posterior tibial nerve at the ankle and pain or paraesthesia in the sole of the foot $(5,6)$. These entrapment neuropathies are recognizable clinically and can be confirmed with use of electrodiagnostic testing $(5,6)$.

Through an extensive literature search by means of the MEDLINE consultation, we have identified different reports of CTS described in HIV-infected patients.

Sclar has hypothesized that carpal tunnel syndrome may be a metabolic consequence of protease inhibitor use (7). In this article, four cases of carpal tunnel syndrome were reported in women aged 35-43 years; these patients had been treated with protease in- hibitors in combination with nucleoside analogues for at least one year and they had been diagnosed between 1 and 4 years earlier with HIV infection. The author has postulated that protease inhibitor use may have led to weight gain predisposing these patients to median nerve entrapment or that metabolic abnormalities associated with protease inhibitors use may lead to the deposition of myxedematous material in the median nerve in these cases (7).

In an Italian study, two patients with HIV infection with confirmed tunnel carpal syndrome while on prolonged HAART have been described (8). The authors stressed the concept that these patients developed a CTS in absence of presumed risk factors for entrapment neuropathies and metabolic abnormalities related to protease inhibitorcontaining HAART (8).

Data obtained from another study by Asensio et al. do not support the hypothesis that the incidence of carpal tunnel syndrome in HIV-positive patients is superior to that in general population (9). These authors have suggested that HAART is not involved in the development of carpal tunnel syndrome. In this study, the authors recognized six patients that fulfilled the criteria for a diagnosis of carpal tunnel syndrome; these six patients represented $0.9 \%$ of all HIV patients and $0.8 \%$ of HIV patients who were receiving HAART in their unit. In these patients there was evidence of other risk factors for carpal tunnel syndrome, unrelated to HIV infection. All patients had intensive use of the hands in their jobs and one of them suffered from hypothyroidism. Two patients had never received antiretroviral therapy and in another carpal tunnel syndrome began before initiating HAART. None of four patients treated with HAART developed lipodystrophy (9).

Before the introduction of HAART, carpal tunnel syndrome was rarely reported among the neurological manifestations of HIV infection $(10,11)$.

In the study by Wooley et al., which described neurological manifestations among 94 outpatients over a six months period from August 1993 until January 1994 in 
Australia, neurological abnormalities noted included carpal tunnel syndrome in one patient (11).

In a large series of children undergoing nerve conduction studies in 1995, idiopathic median nerve compression at the carpal tunnel was found in less than $0.5 \%$ (11).

In another study, Floeter et al. surveyed the charts of children with HIV infection referred to the EMG laboratory at the National Institutes of Health in Bethesda between 1989 and 1995, for evaluation of suspected peripheral neuropathy (12). Among twelve children who had an abnormal nerve conduction study, the findings suggested a median nerve compression at the carpal tunnel in three children aged 4.5, 7, and 14.5 years. Only the two younger children had received antiretroviral treatment with $\mathrm{zi}^{-}$ dovudine (AZT) plus didanosine (ddI) or dideoxycitosine (ddC). Median motor and sensory studies in these children showed prolonged sensory latencies and mildly prolonged residual motor latencies. These children continued to be followed clinically, and none developed multifocal motor neuropathy in the subsequent follow-up periods of 2 to 3 years. During the follow-up period the nerve conduction study abnormalities persisted (12).

A 48-year-old woman who presented with CTS secondary to tuberculous tenosynovitis and who subsequently proved to have HIV infection was documented in 1999 (13).

CTS has also been described as potential side effect in HIV-infected patients treated with the recombinant growth hormone used for the treatment of weight-losing HIVinfected patients (14), and in patients with buffalo hump or other manifestations of the HIV-associated adipose redistribution syndrome associated with $\operatorname{HAART}(15,16)$. Interleukin-2 (IL-2), a cytokine extensively used in the treatment of cancer patients and used in the past years as adjuvant therapy in patients with HIV infection, can produce focal entrapment of the median nerve at the wrist by mediating the inflammatory response and causing interstitial edema, which reverses with drug withdrawal (17). Symptoms usually occur during or shortly after IL-2 infusion and resolve after cessation of therapy with conservative management.

Reyes-Corcho et al. (18) reported the first clinical case of CTS in a Cuban HIV-infected male patient who had no ART. The 35 year-old male patient was admitted due to pain, numbness and tingling in the right upper limb, primarily in the hand and wrist for three months, without preceding opportunistic illnesses, and with CD4+ T cell count over 200 cells $/ \mathrm{mm}^{3}$. There was complete resolution of symptoms with specific surgical treatment (18).

The exact role of HIV in the etiopathogenesis of CTS remains obscure, and other concomitant factors such as nutritional deficiencies, metabolic disorders, drug side effects and HCV infection may be responsible for CTS in these patients.

$\mathrm{HCV}$ infection exhibits a wide range of extrahepatic complications and numerous HCV patients suffer peripheral neurological manifestations. Patients with chronic HCV infection reported a wide variety of rheumatological and neurological manifestations, impairing their quality of life, with considerable frequency (19). The vascular deposition of immune complexes, cryoglobulins, as well as autoantibodies, and/or HCV RNA containing cryoglobulins may be a pathogenic mechanism responsible for nervous system dysfunction. Alternatively, direct viral invasion and perivascular inflammation may be another factor that severely affects the nervous system (19).

A case study of 19 consecutive patients presenting with rheumatic manifestations and who were subsequently found to have chronic hepatitis C, documented CTS in 8 patients (20).

In a study evaluating the presence of symptoms related to rheumatological manifestations in 114 patients with $\mathrm{HCV}$ infection and cryoglobulinemia, CTS was found in 7 $(6 \%)$ patients $(21)$.

In a study to determine the prevalence of peripheral neuropathies in 78 patients with $\mathrm{HCV}$ in the Brazilian Amazon, the researchers observed $5.1 \%$ of CTS (22). CTS was reported in $42.6 \%$ of sixty pa- 
tients with extra-hepatic manifestations of chronic HCV infection generally associated with genotype $2 c$ (23).

Among 40 patients suffering from CTS and attending Al-Azhar University Hospitals, $13 \%$ (32.5) patients showed positive HCV markers after exclusion of other possible cases of CTS during preoperative investigation (24).

CTS was diagnosed in 40 patients $(10.4 \%)$ in a study that evaluated CTS as a dialysisrelated amyloidosis manifestation in 386 patients on maintenance haemodialysis.

The authors have speculated that this relationship may be due to stimulation of the liver by the inflammatory process of producing beta-2-microglobulin (BMG), and this in turn plays an essential role in the pathogenesis of dialysis-related amyloidosis $(25)$.

Deposition of high BMG levels in tissue as amyloid appeared, in the past, to be associated with development of CTS, in addition to long term dialysis and age. To prevent development and progression of CTS, reduction of the blood BMG level through improved techniques for BMG removal may be of most importance (26).

In a recent electrophysiological study to investigate the existence of peripheral and optic neuropathies in thirty consecutive patients in a chronic hepatitis outpatient clinic, altogether nine patients exhibited CTS (27).

In view of the literature data, the incidence of CTS in HIV-positive patients appears to be similar to that observed in the general population, however multiple and often concurrent risk factors for the development of CTS may be observed in these patients, making very difficult to understand the precise etiologic and pathogenetic mechanism of CTS. Physician should maintain a high level of clinical suspicion in HIV-positive patients with symptoms indicating carpal nerve suffering, to define timely diagnosis and appropriate therapy. Advanced HIV disease and ART may be considered risk factors for development of CTS, possibly due to an increased inflammatory state and the presence of concurrent $\mathrm{HCV}$ infection.

\section{REFERENCES}

1. Lima ALLM, Godoy AL, Oliveira PRD, et al. Orthopedic complications in HIV patients. Rev Brasil Ortop. 2009; 44: 186-90.

2. Walker UA, Tyndall A, Daikeler T. Rheumatic conditions in human immunodeficiency virus infection. Rheumatology. 2008; 47: 952-9.

3. Mastroianni A, Cancellieri C, Allegrini F, Pignatari S. Sindrome del Tunnel Carpale ed infezione da HIV: descrizione di 3 casi. XXVIII Congresso Nazionale A.M.O.I., Venezia 4-7 Dicembre 2000. Libro degli Abstracts [Abstract Pagina 256].

4. Mastroianni A, Cancellieri C, Allegrini F, Pignatari S. Carpal Tunnel syndrome in HIVpositive patients coinfected with $\mathrm{HCV}$. $8^{\text {th }} \mathrm{Eu}-$ ropean Conference on Clinical Aspects and Treatment of HIV Infection, Athens, Greece, 28-31 October 2001. Abstracts Book pag. 224 [Abstract P 374].

5. McArthur JC. Neurologic manifestations of AIDS. Medicine. 1987; 66: 407-36.

6. Harrison MJG, McArthur JC. Peripheral nerve disease. In: Harrison MJG, McArthur JC, eds. AIDS and Neurology. Edinburgh: Churchill Livingston; 1995; 87-108.

7. Sclar G. Carpal tunnel syndrome in HIV-1 patients: a metabolic consequence of protease inhibitor use? AIDS. 2000; 14: 136-8.

8. Manfredi R, Calza L, Chiodo F. Carpal tunnel sindrome in HIV-infected patients treated with highly active antiretroviral therapy: other case reports. Rheumatol Int. 2001; 21: 81-3.

9. Asensio O, Arranz Caso JA, Rojas R. Carpal tunnel sindrome in HIV patient. Carpal tunnel sindrome in HIV patients? AIDS. 2002; 16 : 948-50.

10. Wooley I, Faragher M, Spelman D. Neurological manifestations of HIV in an outpatient clinic including a case control study of factors with peripheral neuropathy. Annu Conf Australas Soc HIV Med. 1994; 6: 271 [unnumbered poster].

11. Brouwers P, Tudor-Williams G, De Carli C, et al. Relation between stage of disease and neurobehavioral measures in children with symptomatic HIV disease. AIDS. 1995; 9: 713-20.

12. Floeter MK, Civitello LA, Everett CR, et al. Perpheral neuropathy in children with HIV infection. Neurology. 1997; 49: 207-12.

13. Andersson MI, Wilcox PA. Tuberculous tenosynovitis and carpal tunnel syndrome as a presentation of HIV disease. J Infect. 1999; 39: 240-1.

14. Cominelli S, Raguso CA, Karsegard L, et al. Weight-losing HIV-infected patients on recombinant human growth hormone for $12 \mathrm{wk}$ : a national study. Nutrition. 2002; 18: 583-6.

15. James JS. Successful treatment of "buffalo hump" with growth hormone. AIDS Treat News. 1998; 298: 5-6. 
16. Torres R, Unger K. The effect of recombinant human growth hormone on protease-inhibitor-associated fat maldistribution syndrome. $6^{\text {th }}$ Conf Retroviruses Opportunistic Infect 1999;194 [abstract no. 675].

17. Puduvalli VK, Sella A, Austin SG, Forman AD. Carpal tunnel sindrome associated with interleukin-2 therapy. Cancer. 1996; 77: 118992.

18. Reyes-Corcho A, Barrueta-Reyes D, BouzaJiménez Y, et al. Carpal tunnel syndrome and HIV infection. A case report and literature review. Infect Dis Rep. 2009; 1: e2.

19. Mathew S, Faheem M, Ibrahim SM, et al. Hepatitis $C$ virus and neurological damage. World J Hepatol. 2016; 8: 545-56.

20. Lovy MR, Starkebaum G, Uber. Hepatitis C infection presenting with rheumatic manifestations: a mimic of rheumatoid arthritis. J Rheumatol. 1996; 23: 979-83.

21. Leone N, Pellicano R, Ariata Maiocco I, et al. Mixed cryoglobulinaemia and chronic hepatitis $\mathrm{C}$ virus infection: the rheumatic manifestations. J Med Virol. 2002; 66: 200-3.

22. Gomes I, Daniel Nora DB, Marquezini NC, et al. Peripheral neuropathy in patients with hep- atitis virus $\mathrm{C}$ infection in the Amazon region. Arq Neuropsiquiatr. 2006; 64: 600-2.

23. Giannitti C, Morozzi G, D'Alfonso S, et al. Viral genotype and HLA class II alleles influence on extra-hepatic manifestations of chronic HCV infection. Reumatismo. 2008; 60: 192-8.

24. Eleowa S, Emam H, Essa AS. Is there a correlation between carpal tunnel syndrome and hepatitis C virus infected patients? (Preliminary Report). Egypt J Plast Reconstr Surg. 2012; 36: 83-6.

25. Kopeć J, Gądek A, Drożdż M, et al. Carpal tunnel syndrome in hemodialysis patients as a dialysis-related amyloidosis manifestation incidence, risk factors and results of surgical treatment. Med Sci Monit. 2011; 17: 505-9.

26. Shin J, Nishioka M, Shinko S, et al. Carpal tunnel syndrome and plasma beta-2- microglobulin concentration in hemodialysis patients. Ther Apher Dial. 2008; 12: 62-6.

27. Köşkderelioğlu A, Ortan P, Ari A, Gedizlioğlu M. Screening for electrophysiological abnormalities in chronic hepatitis $\mathrm{C}$ infection: peripheral neuropathy and optic neuropathy. Nöro Psikiyatri Arşivi. 2016; 53: 23-7. 